\title{
Coronary Artery Calcium Score: A Review
}

\author{
Abbas Arjmand Shabestari ${ }^{1,2, *}$ \\ ${ }^{1}$ Radiology Department, Modarres Hospital, Shahid Beheshti University of Medical Sciences, Tehran, IR Iran \\ ${ }^{2}$ Advanced Diagnostic and Interventional Radiology Research Center (ADIR), Tehran, IR Iran \\ 'Corresponding author: Radiology Department, Modarres Hospital, Shahid Beheshti University of Medical Sciences, Tehran, IR Iran. Phone: +98-21-22083111, +98-21-88336335, Fax: \\ +98-2122074101, E-mail: abarjshabestari@yahoo.com; abbas_arjmand@sbmu.ac.ir \\ Received: September 1, 2013; Revised: September 25, 2013; Accepted: September 27, 2013
}

\begin{abstract}
Context: Coronary artery disease (CAD) is the foremost cause of death in many countries and hence, its early diagnosis is usually concerned as a major healthcare priority. Coronary artery calcium scoring (CACS) using either electron beam computed tomography (EBCT) or multislice computed tomography (MSCT) has been applied for more than 20 years to provide an early CAD diagnosis in clinical routine practice. Moreover, its association with other body organs has been a matter of vast research.

Evidence Acquisition: In this review article, techniques of CACS using EBCT and MSCT scanners as well as clinical and research indications of CACS are searched from PubMed, ISI Web of Science, Google Scholar and Scopus databases in a time period between late 1970s through July 2013 and following appropriate selection, dealt with. Moreover, the previous and ongoing research subjects and their results are discussed.

Results: The CACS is vastly applied in early detection of CAD and in many other research fields.

Conclusions: CACS has remarkably changed the screening techniques to detect CAD earlier than before and is generally accepted as a standard of reference for determination of risk of further cardiac events.
\end{abstract}

Keywords: Atherosclerosis; Tomography, X-Ray Computed; Arteries; Coronary Artery Disease; Calcium

\section{Context}

Coronary artery disease (CAD) is the foremost cause of death in many countries throughout the entire world. Albeit more common in Western countries, it seems to be increasing in frequency through the non-industrialized countries, as well, likely reflecting a change in their inhabitants' lifestyles (1).

Over the past decades it has been well demonstrated that coronary artery atherosclerotic plaques are the main causes of CAD. While in progression, the coronary artery plaques may contain calcium; hence, it has been suggested that finding of calcified foci at coronary artery walls may indicate CAD and its extent. From the early 1980 s the presence of coronary arterial mural calcified foci was found to be related to CAD (2-4).

Indeed, Margolis et al. (5) showed the significance of coronary artery calcification in diagnosis of CAD and in determining its prognosis. In their study the calcified foci were detected in coronary arteries area at fluoroscopic assessment of 800 patients and their impact on future cardiac events was evaluated $(5,6)$.
For the first time in 1979 Guthaner and her colleagues demonstrated the ability of computed tomography (CT) to find coronary artery calcifications $(7,8)$. Thereafter, it was gradually demonstrated that CT is much more sensitive than fluoroscopy in calcium detection $(9,10)$. On the other hand, beating heart has always been a problem for imaging, requiring faster image acquisition to improve the temporal resolution, so electron beam CT (EBCT) scanners also known as ultrafast CT scanners were further developed. CT scanner developments resulted in ability to find smaller coronary calcified foci enabling the researchers to make reproducible quantitative measurements in coronary artery calcium scoring (CACS) (11-13).

\section{Evidence Acquisition}

The terms "coronary", "calcium", "score" and "computed tomography" were searched among the databases of PubMed, ISI Web of science, Scopus and Google Scholar to find relevant data from late 1970s (introduction of body CT) through July 2013 and totally 1023 published resources were found. Irrelevant and repetitive resources

Implication for health policy/practice/research/medical education:

This review article deals with coronary artery calcium scoring which is of remarkable interest to healthcare policymakers, cardiologists, radiologists, cardiac surgeons, medical researchers as well as people working in field of medical education.

Copyright (C) 2013, Iranian Red Crescent Medical Journal; Published by Kowsar Corp. This is an open-access article distributed under the terms of the Creative Commons Attribution License, which permits unrestricted use, distribution, and reproduction in any medium, provided the original work is properly cited. 
$(n=926)$ were excluded based on their titles and/or Abstract contents and a few $(n=8)$ others (comprising unpublished ones until then) were included in study. Ultimately, 105 references were assessed.

\section{Results}

\subsection{Technical Aspects}

The EBCT scanners provided electron ray targeting a ring anode around the patient's body and hence, obviated the need for presence of a mechanically-rotating $\mathrm{X}$-ray tube, resulting in remarkably faster image acquisition and thereby, improving the temporal resolution $(11,12,14)$. For the first time EBCT which could provide appropriate temporal resolution for cardiac imaging was used for CACS $(15,16)$. However, EBCT was not applicable in imaging of most other body organs, since its high noise - low signal-to-noise ratio (SNR) - resulted in poor image quality, while its improved temporal resolution had not any positive impact on imaging of other non-moving or less-moving body organs. The aforementioned high image noise leads to inappropriate image quality and hence, EBCT is usually not used in coronary CT-angiography (CCTA). Moreover, EBCT is more expensive than most routinely used CT scanners and its dimensions are larger, requiring remarkably more roomy space for scanner to be installed. Using EBCT the parameters for CACS were as follows: longitudinal (z-axis) scan coverage from tracheal carina to diaphragm during breath hold, no contrast agent injection, $3 \mathrm{~mm}$ slice thickness, electrocardiography (ECG)-gated sequence (non-spiral) mode acquisition at $80 \%$ of R-R interval, 512 X 512 matrix size, $130 \mathrm{kVp}$, current of $630 \mathrm{~mA}$ and $100 \mathrm{mSec}$ acquisition time (15). As pointed out before, the EBCT scanners were merely applied to determine CACS. Introducing multislice CT (MSCT) scanners from 1998 and their substantial developments through the last 15 years has led to their gradual supersession of the EBCT (17). Given the remarkably better spatial resolution of MSCT scanners, nowadays ECG-synchronized CCTA is widely used and in most cases, CACS is performed as a conjunct and usually prior to CCTA using MSCT (18-21). The advancements of MSCT scanners led to improvement of spatial resolution and SNR as well as less noisy images in comparison with EBCT. From their introduction, the temporal resolution of various MSCT generations has gradually improved from $500 \mathrm{mSec}$ in first 4-slice MSCT scanners to less than $100 \mathrm{mSec}$ in recently introduced ones (22). The improved imaging quality has led to extended use of MSCT in CCTA. The same holds true about CACS and hence, nowadays MSCT is much more widely used than EBCT for CACS (23). A high image quality is obtained using MSCT scanners when utilizing retrospective ECG-gating technique to find the most appropriate phase with least motion artifact $(24,25)$. Moreover, Horiguchi et al have shown that using prospective ECG-triggered scan at $45 \%$ of R-R inter- val in a 64-slice MSCT scanner provides a constantly high image quality irrespective of heart rate, body mass index or background noise level (26). In a study by Groen et al both 64-slice MSCT scanner and dual-source CT scanner were compared with EBCT, revealing more association of dual-source CT with EBCT in comparison with 64-slice MSCT. This higher correlation was more perceptible when using thinner slices and particularly $0.6 \mathrm{~mm}$ slices (27). It had been shown by Ulzheimer and Kalender (28) that image quality of 4-slice MSCT scanners for CACS was equal or even better than EBCT for cardiac imaging. Horiguchi et al found a high agreement between 16-slice MSCT scanner and EBCT in CACS (29). The inter-scan variability was demonstrated to be less than EBCT when performed by MSCT scanners in a study by Kopp et al. (30). Assessment of CACS using thin-slice $(0.5 \mathrm{~mm})$ 320-slice scanner by Van der Bijl (31) showed that small calcified plaques detection is more accurate when compared with standard thicker (3 mm) slices (31). When compared with standard $3 \mathrm{~mm}$ thickness EBCT technique, various both thicker and thinner slices were applied to determine CACS. Thick-slice (5-6 $\mathrm{mm}$ ) EBCT scan was used by Detrano et al. (32) in order to shorten scanning time and reduce the background noise, which demonstrated similar scores and prognostic value in CAD. On the other hand, there are a few studies that showed applicability of thinner than $3 \mathrm{~mm}$ slices using MSCT scanners (which reveal less noise than EBCT images) in CACS $(27,31,33)$. Not only the slice thickness is of paramount importance, but also the reconstruction interval of slices has its impact on CACS. The effect of using varying reconstruction intervals in 16-slice MSCT scanner is assessed by Schlosser et al. (34); while recently, it has been demonstrated to have a significant importance in CACS accuracy using dual-source CT scanners, particularly in cases of low calcium score (24). Frequent use of CACS protocol as a routine adjunct of CCTA leads to increase of patient's radiation dose. The radiation dose associated with CAC scoring is small and ranges from 0.9 to 2.4 milliSievert ( $\mathrm{mSv}$ ) in different multislice CT scanners (35). In some cardiac CT protocols, the radiation doses are estimated to be higher than $10 \mathrm{mSv}$ (35-37) leading to a small but measurable increase in the risk of radiation-induced cancer and hence this fact should be concerned in cases that CACS and repeated exams are used as a widespread population screening (36). Some have proposed that while CCTA is able to assess the CAD extent, CACS may not be necessary to be carried out; nevertheless, it has been shown that whenever the density of intraluminal contrast is increased, coronary mural calcified plaques may not be detected and hence, resultant from their similar attenuation values may be missed (31). Image densities are shown to be variable based on CT scanner type as well as patient's body habitus by Nelson et al and using calibration phantoms has been demonstrated to reduce the inter-scan variability in calcium density measurements (38). An automatic attenuation-based tube current adaptation technique was proposed by Mühlenbruch et al in a 
16-slice MSCT scanner to reduce patient's radiation dose and image noise in CACS (39).

\subsection{Agatston Score}

The first practically applicable quantitative CACS protocol was introduced by Arthur Agatston and his colleagues ( 15 )in 1990 and has still remained the standard method in CACS. Any structure which had densities of 130 Hounsfield units (HU) or more and having an area of $1 \mathrm{~mm}^{2}$ or more was segmented as calcified focus and those foci overlying the anatomic site of coronary arteries were considered to represent calcified plaques. Using an area of at least $1 \mathrm{~mm}^{2}$ - comprising of at least 2 pixels - ensured one not including single pixel - which represents image noise - in measurements. In each segmented calcified focus, based on its peak density, a density score of 1 through 4 was assigned. The stratified density scores 1, 2, 3 and 4 represented the highest densities 130-199 HU, 200-299 $\mathrm{HU}, 300-399 \mathrm{HU}$ and $\geq 400 \mathrm{HU}$, respectively. The most important determining factors in calculating calcium score of each plaque were the measured area of each calcified plaque and its density. The total Agatston score (AS) of each individual was calculated by summing the scores of every calcified focus through all of the coronary arteries (15). Figure 1 shows the Agatston score measurement technique and its resultant values demonstrated in a dedicated table.
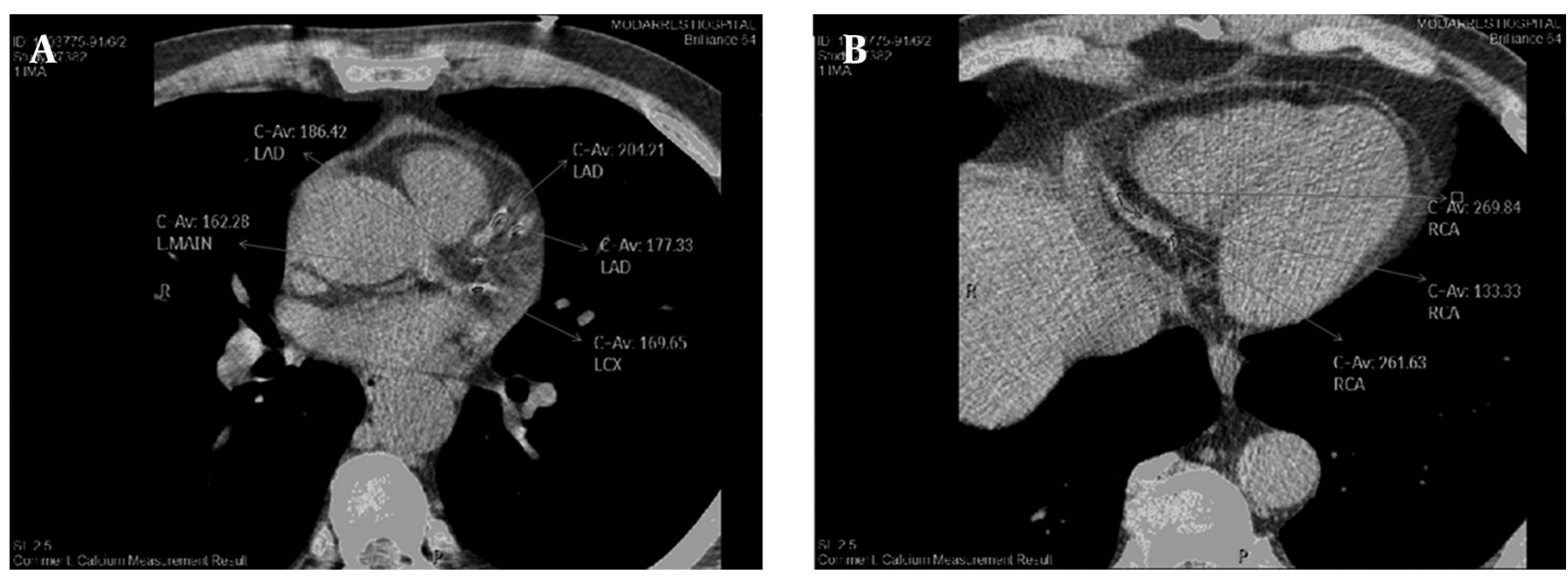

\begin{tabular}{|c|c|c|c|c|c|}
\hline C & \multicolumn{5}{|c|}{ Scoring Results : Mass Score Protocol } \\
\hline & L.MAIN & LAD & LCX & RCA & Total Coronaries \\
\hline Score & 6.67 & 26.98 & 1.24 & 34.32 & 69.21 \\
\hline \#ROI's & 2 & 7 & 1 & 14 & 24 \\
\hline Volume (cu.mm.) & 40.16 & 159.71 & 9.23 & 202.63 & 411.73 \\
\hline
\end{tabular}

Figure 1. Coronary calcium score non-contrasted ECG-gated computed tomographic (CT) views of coronary arteries demonstrate presence of multiple calcified plaques through the anatomic territory of left main (L.MAIN) coronary artery and proximal segments of left anterior descending (LAD) and left circumflex (LCX) coronary arteries and their branches (A) and distal segment of right coronary artery (RCA) (B). The measurement table (C) provided by CT workstation demonstrates the calcium score of each coronary artery and their total score based on Agatston technique in the first row, the number of assigned calcified plaques in each territory and their total number in the second row and measured area of the corresponding plaques (according to square millimeters) in the third row. The measured total coronary calcium score (389.57 Agatston Units) in this 66-year old man equals to $77 \%$ for that particular gender (male) and age range (65-69 years) according to an available database calculated and shown in the last row.

\subsection{Volume Score}

Since AS required a relatively complex measurement technique, in an effort to simplify the coronary calcium measurement and increase its reproducibility, "volume score" was first introduced by Callister et al. (40) simply calculated based on segmented calcified plaque area and number of slices containing each of those plaques. The volume score was expressed in milliliters. No peak density measurement is used in volume score calculation and hence, its inter-scan variation is less than AS. Nonetheless, this variability increases in high calcium scores, so Hokanson et al. (41) used the square root of volume score in order to decrease this variation. Analysis of AS and volume score has been shown to be similar in reference data establishment of age-sex percentile ranking $(42,43)$. 


\subsection{Mass Score}

In 2002 Hong et al. (33) introduced a technique to measure "mass score" of calcified coronary plaques which measures the absolute real mass of coronary calcium. Albeit it may be considered more accurate and more reproducible than Agatston and volume scores, it requires a phantom containing different concentrations of calcium hydroxyapatite (CaHA) placed beneath the patient's thorax in order to calibrate the segmented coronary calcium and hence, is more complicated than former ones in hardware $(33,44)$. The absolute score is expressed as milligrams of CaHA in this stratification. Despite presence of a few papers revealing its weaknesses, it is shown that mass score may be even more reproducible than Agatston or volume scores in high scores, so that Ulzheimer and Kalender in 2003 (28) suggested changing from a particular Hounsfield Unit threshold to a calcium equivalent expressed as mg of $\mathrm{CaHA} / \mathrm{cm}^{3}$ to calculate CACS. Based on their suggestion, this new measurement would not differ among varying CT scanners (28). Figure 2 demonstrates the mass score measurement technique and corresponding calculated values shown in a dedicated table.
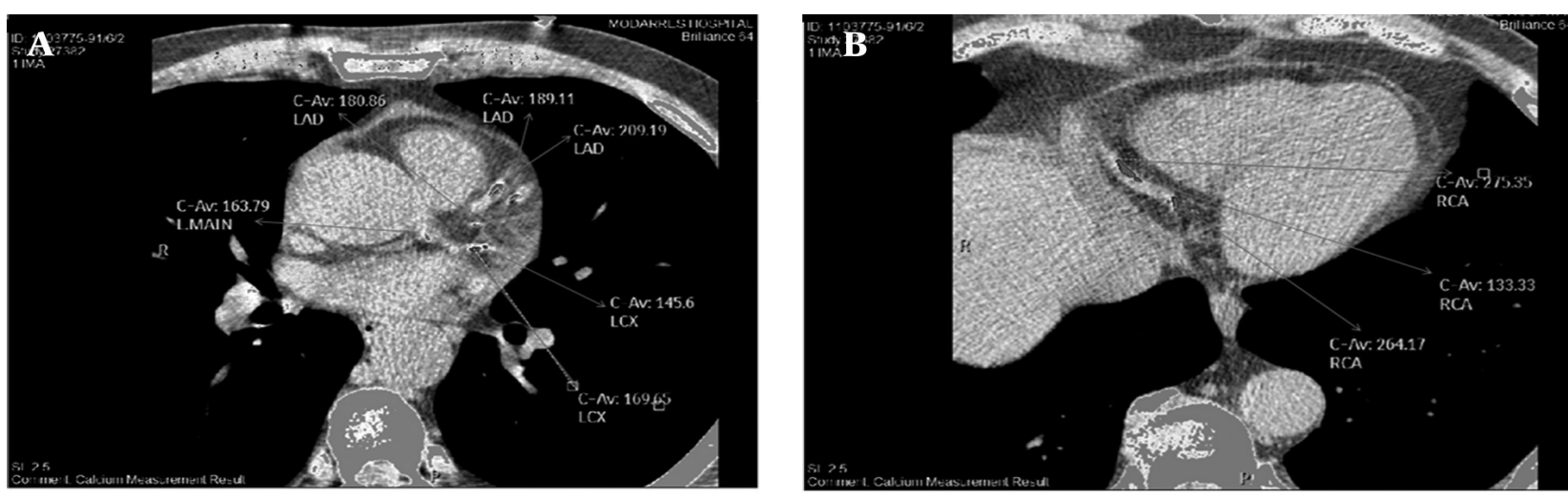

\begin{tabular}{|c|c|c|c|c|c|}
\hline $\mathbf{C}$ & \multicolumn{5}{|c|}{ Scoring Results : Agatston Score Protocol } \\
\hline & L.MAIN & LAD & LCX & RCA & Total Coronaries \\
\hline Score & 35.54 & 159.24 & 7.54 & 187.25 & 389.57 \\
\hline \#ROI's & 2 & 8 & 3 & 16 & 29 \\
\hline AreaSq (sq.mm.) & 15.69 & 62.22 & 5.35 & 79.21 & 162.48 \\
\hline
\end{tabular}

Figure 2. Coronary calcium score non-contrasted ECG-gated computed tomographic (CT) views of coronary arteries of the same patient as in Fig-1 demonstrating presence of multiple calcified plaques through the anatomic territory of left main (L.MAIN) coronary artery and proximal segments of left anterior descending (LAD) and left circumflex (LCX) coronary arteries and their branches (A) and distal segment of right coronary artery (RCA) (B). The measurement table (C) provided by CT workstation demonstrates the mass calcium score of each coronary artery and their total score based on Mass Score protocol in the first row, the number of assigned calcified plaques in each territory and their total number in the second row and measured area of the corresponding plaques (according to square millimeters) in the third row. The total coronary calcium Mass Score is measured to be 69.21 in this individual.

There is no published absolute quantification reference standard of plaque burden. Nevertheless, standard for risk stratification in percentile for Agatston, volume and mass scores was published by Rumberger and Kaufman in 2003 (42) as a large-scale (in 11,490 patients) research. The mass score is considered to be a reliable CACS technique both in research and in clinical routine practice.

\subsection{Calcium Coverage Score}

More recently in 2008, Brown et al. (45) established another technique called calcium coverage score. Applying calcium coverage score in a Multi-Ethnic Study of Atherosclerosis (MESA) associated with its correlation with AS and mass score, the coronary arteries percentage involved in calcification was called calcium coverage score. Calcium coverage score was shown to be accompanied by hypertension and diabetes as well as dyslipidemia (45).

\subsection{Validation and Clinical Applications of CACS}

Many studies were carried out based on Agatston method trying to use this technique to stratify patients' CAD extent, one of the first ones being Rumberger's study. Rumberger and his colleagues provided a stratified guideline according to CACS in order to determine the coronary plaque burden and resultant CAD severity shown in Table 1 (46). Hoff et al. (16) used the same EBCT technique in more than 35,000 individuals to determine the AS percentiles in each gender and each of the categorized age groups. Another study based on the same agegender percentiles have shown the significantly higher CAD risk of those above the 75th percentile in comparison with those below the 25th percentile (47). In 2003, Rumberger \& Kaufman (42) using Agatston, mass and volume CACS techniques suggested another risk stratification model for CAD in more than 11,000 individuals. 
Over the past years CACS validity has been assessed in many studies. Since it was previously shown that there is poor correlation between the EBCT findings of coronary calcium and the luminal narrowing severity in coronary catheter angiography, Rumberger et al. (48) made a comparison of EBCT measured calcified atherosclerotic plaque area with plaque area measured in histopathologic findings of 13 heart autopsy exams. They figured out that there was a close association between plaque extent and coronary calcification area. The discrepancies could be described by the presence of non-calcified plaques and the so called "positive remodeling" of coronary arter- ies (48).

Guerci et al. (49) showed that the AS has a remarkably significant correlation with coronary narrowing severity and therefore, suggested that CACS could represent CAD extent. Rumberger and his colleagues (50) presented a CACS cut-off point to predict the severe luminal narrowing. They proposed AS of 371 as predictor of more than $70 \%$ narrowing in as a minimum one of the coronary arteries. Similarly, Moser et al suggested 400 AS could be considered an edge score for a further requirement to carry out nuclear myocardial perfusion scan in asymptomatic patients (51).

\begin{tabular}{lll}
\hline \multicolumn{2}{l}{ Table 1. The first Rumberger guideline based on Agatston score using Electron Beam Computed Tomography $($ EBCT $)(47)$} \\
\hline Calcium Score & Plaque Burden & Clinical Interpretation \\
\hline $\mathbf{0}$ & None & Very low risk of cardiovascular disease \\
& & Likelihood of coronary artery disease presence $<5 \%$ \\
$\mathbf{1 - 1 0}$ & Minimal & Negative examination \\
$\mathbf{1 1 - 1 0 0}$ & Mild & Significant coronary artery disease very unlikely \\
$\mathbf{1 0 1 - 4 0 0}$ & Moderate & Likely mild or minimal coronary stenosis \\
Over 400 & Extensive & Moderate non-obstructive coronary artery disease highly likely \\
\hline
\end{tabular}

According to Mendoza-Rodriguez and colleagues using 64-slice MSCT, volume score was significantly correlated with flow-limiting CAD (52).

Shaw and colleagues assessed coronary calcium as a risk in all-cause mortality estimate and included 10,377 asymptomatic subjects proving that calcification provides non-dependent information additionally to Framingham risk factors (53).

Comparing with prevalence of CAD in 17,967 asymptomatic individuals, Cheng et al. (54) realized that there was an increased risk of CAD at all levels higher than 95 AS. Guerci and colleagues in a study of of 290 subjects suggested 80 as the cutoff AS value in forecasting the augmented CAD likelihood. Based on these findings, the absolute AS value revealed its potential as a sensitive method for CAD screening (55). Using MSCT, Shabestari et al showed a moderate-to-good agreement between CACS of more than 100 AS and significant coronary stenosis (56). Through the recent years the clinical clarification of a "zero" score has been subject of major debate. Wexler et al. (57) described that a zero calcium score almost with certainty implied CAD absence. Nevertheless, it should be reminded that absence of coronary calcified plaque does not exclude presence of soft plaque (and resultant acute coronary syndrome). Shemesh et al. (58) declared that there was a contradiction as minimal CACS could characterize those who may present with acute symptoms, whereas presence of diffusely distributed and high-density calcified plaques could be associated with chronic coronary events. Thompson and Stanford commented that the zero calcium might exclude significant narrowing but could not rule out the CAD and suggested that if there is not any risk factor, there won't be any requirement for further diagnostic procedures (59). Knez et al. (60) demonstrated that calcified plaque absence could very precisely exclude significant CAD in individuals in an age group higher than 50 years. On the other hand, Ergun et al showed that there were a substantial amount of subjects who had zero CACS and their CCTA revealed CAD (61). Actually, importance of absent calcified plaque is currently considered to be weaker than that before. Sometimes clinically important soft plaques were detected in CCTA of patients who had no coronary calcium. Nonetheless, Uretsky et al. (62) reported that non-calcified plaques were hardly accompanied by significant stenoses, if ever. Church and colleagues (63) proposed that absence of calcium demonstrates a remarkably limited CAD likelihood. As Grayburn has pointed out in his article, it is important to assess the CAC score in the clinical context before further tests are recommended for patients (64). The well-known Framingham risk score enables prediction of cardiac events in asymptomatic individuals and is estimated according to age, gender, total serum cholesterol level, high-density lipoprotein (HDL) cholesterol level, history of smoking, and systolic blood pressure. This score is denoted as 10-year risk score for the estimation of CAD events likelihood. Nonetheless, growing evidences exist which demonstrate these risk stratification techniques have significant limitations as guidance for treatment of each individual. Based on the question of whether CACS is indicated for screening asymptomatic patients at Framingham intermediate risk for CAD the guidelines differ, however, CACS for symptomatic patients with known CAD is generally accepted 
to be not helpful. The main aim of CACS in asymptomatic persons is to refine the risk assessment to determine whether preventive measures have to be intensified, not finding persons with symptomatic coronary stenosis (23). In those people who are asymptomatic, absence of calcified plaque is accompanied by a remarkably mild ( $<1 \%$ in each year) risk of main coronary events through the upcoming 3-5 years, while presence of high CACS in asymptomatic individuals may increase this risk up to 11-fold (65). A MESA paper revealed that there is a remarkable variation in CACS measured in various ethnicities. Nevertheless, CACS had an additive importance in their prognosis determination so that in those people who had AS $>100$, in comparison with those who had not any calcified plaque, the prevalence of coronary events may show a 7-fold increase (66). CACS is not recommended for screening of individuals who are symptomatic as calcified plaques only have marginal relation to the extent of narrowing and the significance of absent or low CACS in symptomatic patients remains unclear (21). Symptomatic patients should be referred for CCTA to determine the CAD severity and there is no significant incremental value of CACS beyond the CCTA prognostic information in symptomatic patients (67).

\begin{tabular}{|c|c|c|c|}
\hline Study Authors & Year & Study Cohort & Results $^{\mathrm{a}}$ \\
\hline Thompson and Stanford (59) & 2001 & Not Applicable & Exclusion of significant CAD likelihood in CACS $=0$ \\
\hline Shemesh et al. (58) & 2003 & 50 & Low CACS characterized patients with acute coronary events \\
\hline Knez et al. (60) & 2004 & 2,115 & $\begin{array}{l}\text { Very accurate in obstructive CAD exclusion in subjects }>50 \text { years } \\
\text { old }\end{array}$ \\
\hline Church et al. (63) & 2007 & 10,746 & Very low CAD risk in the intermediate term \\
\hline Alkram et al. (71) & 2008 & 210 & $\begin{array}{l}\text { CACS is better in asymptomatic subjects, especially in patients }< \\
45 \text { years to exclude obstructive CAD }\end{array}$ \\
\hline Cademartiri et al. (72) & 2010 & 279 & $\begin{array}{l}\text { Prevalence of significant } \mathrm{CAD} \text { was not negligible in asymptomatic } \\
\text { patients with } \mathrm{CACS}=0\end{array}$ \\
\hline Ergun et al. (61) & 2010 & 883 & $\begin{array}{l}\mathrm{CACS}=0 \text { patients had positive CTA findings, especially when risk } \\
\text { factors exist }\end{array}$ \\
\hline Gottlieb et al. (73) & 2010 & 291 & $\begin{array}{l}\text { Frequent occurrence of total coronary occlusion in CACS }=0 \\
\text { patients }\end{array}$ \\
\hline Uretsky et al. (62) & 2011 & 1,119 & $\begin{array}{l}\text { CACS }=0 \text { is rarely accompanied by hemodynamically significant } \\
\text { CAD }\end{array}$ \\
\hline Esteves et al. (74) & 2011 & 206 & $\begin{array}{l}\text { CACS }=0 \text { excluded inducible ischemia in an intermediate risk } \\
\text { group }\end{array}$ \\
\hline Sonowski et al. (75) & 2011 & 166 & $\begin{array}{l}\text { Relatively low incidence of significant coronary stenosis in } \\
\text { CACS }=0 \text { patients }\end{array}$ \\
\hline Alqarqaz et al. (76) & 2011 & 333 & Nearly one in five patients with $\mathrm{CACS}=0$ had non-calcified plaque \\
\hline Villines et al. (77) & 2011 & 5,128 & $\begin{array}{l}\text { In symptomatic patients with a CACS=0, obstructive CAD is pos- } \\
\text { sible and is associated with increased cardiovascular events }\end{array}$ \\
\hline Chen et al. (78) & 2012 & 519 & $\begin{array}{l}\text { Plaques are present in a significant proportion of individuals with } \\
\text { CACS }=0\end{array}$ \\
\hline Morita et al. (79) & 2012 & 2,160 & $\begin{array}{l}\text { If patients are male and elderly even if } \mathrm{CACS}=0 \text { the likelihood } \\
\text { of vulnerable plaque exists especially in the presence of spotty } \\
\text { calcification }\end{array}$ \\
\hline Kim et al. (80) & 2012 & 1,114 & $\begin{array}{l}\text { Prevalence of obstructive } \mathrm{CAD} \text { and adverse cardiac events are not } \\
\text { negligible in symptomatic patients with } \mathrm{CACS}=0\end{array}$ \\
\hline Meyer et al. (81) & 2012 & 121 & $\begin{array}{l}\text { Significant CAD is extremely unlikely in symptomatic Caucasian } \\
\text { patients with an intermediate risk score and } C A C S=0\end{array}$ \\
\hline Büyükterzi et al. (82) & 2013 & 288 & $\begin{array}{l}\text { The frequency of non-calcified plaques is too high to be ignored in } \\
\text { CACS }=0\end{array}$ \\
\hline Cho et al. (83) & 2013 & 4,491 & $\begin{array}{l}\text { A future risk of exclusive non-calcified plaque in asymptomatic } \\
\text { subjects with CACS }=0 \text { was negligible }\end{array}$ \\
\hline Lee et al. (84) & 2013 & 6,531 & $\begin{array}{l}\text { In asymptomatic subjects with } \mathrm{CACS}=0 \text { presence of non-calcified } \\
\text { plaque was associated with cardiac events }\end{array}$ \\
\hline Mouden et al. (85) & 2013 & 868 & $\begin{array}{l}\text { A CACS }=0 \text { in stable patients at low or intermediate risk excludes } \\
\text { flow-limiting CAD }\end{array}$ \\
\hline
\end{tabular}




\footnotetext{
a Abbreviations: CAD: Coronary artery disease; CACS: Coronary artery calcium score; CTA: Computed tomography angiography
}

Controversies regarding the clinical interpretation and application of "zero CACS" in different risk-stratified cohorts of patients have continued to persist and are summarized in Table 2 . There are some publications which suggest applying the calcium coverage score as a useful filter for CCTA to diagnose noteworthy coronary disease in individuals who present as having chest discomfort. These imply that zero CACS can exclude obstructive coronary disease and hence obviate the requirement of any further assessment using imaging, while those individuals who have higher CACS may further undergo CCTA to determine their stenosis severity. Nonetheless, it should be pointed out that CACS and coronary CTA can be under remarkable influence of CAD pretest likelihood: severe CACS foresees significant CAD while concurrently degrades the CCTA image - because of blooming artifact of calcium - so that CCTA may be excluded in the management of patients with high CACS $(68,69)$. Meng and coworkers (69) demonstrated that a score of more than 400 resulted in significantly unwanted impact on CCTA accuracy even when implemented by dual-source CT. Hence, based on the Asian Society of Cardiac Imaging (ASCI) published appropriateness criteria, the CCTA had an indeterminate appropriateness when the previous CACS in asymptomatic patients was $\geq 400$ (70).

The appropriateness criteria for CCTA published in 2010 by a joint group of some American scientific societies suggested that in symptomatic patients two groups may be considered: a- in case of a calcium coverage score $>400$, the diagnostic influence of CACS on the decision to carry out CCTA was "uncertain" and b- in a calcium coverage score $\leq 400$, the corresponding diagnostic effect of CACS was considered to be "appropriate" (86). Recently, Otton et al showed that in those individuals who have a CACS $>600$, a negative CTCA implied an excellent shortterm outcome and appeared to exclude clinically significant coronary disease (87). Ahn et al. (88) in a group of 253 patients who had CACS of $>400$ found that despite good overall diagnostic accuracy, CCTA was limited by low specificity. Another study showed that the accuracy of CCTA in the presence of a high coronary calcium score may be underestimated (89).

Considering the CACS validity, other clinical applications have been introduced, as well. Over the past years, CACS has been used as standard of reference quantitative technique for diagnosis of atherosclerosis of either coronary arteries or other non-coronary arterial structures. Indeed, calcium scoring methods are also used to calculate the calcification in other body organs, including the cardiac valves like the aortic valve, in a quantitative manner (90).

The CACS was used as a reference for CAD risk. Based on the Rotterdam study, the calcium score might be utilized in re-stratification of elderly who are at intermediate risk in 10-year Framingham score to assign them in high-risk or low-risk clusters with cut-off CACS of 615 and 50 AS, respectively (91). Based on MESA database, Sirineni and coworkers proposed "coronary age" to predict CAD likelihood, formulating it based on ethnic groups and gender, so that AS was applied as an input factor to calculate the "coronary age" of any individual (92).

Not only the CACS has established as a CAD screening test, but also the coronary CTA has significantly improved to determine whether or not the coronary arterial lumen is patent. The CCTA is of more important role than CACS for CAD assessment; therefore, following CACS, patients may undergo CCTA to assess CAD likelihood. Hence, CACS has been considered to be a "gatekeeper" for CCTA (68-70, 86). Colletti et al. (93) in a group of elderly asymptomatic subjects found a remarkable relationship between CACS and forthcoming regional left ventricular wall motion abnormality as another indicator of a likely subclinical ischemic heart disease depicted in cardiac magnetic resonance imaging (MRI). Stolzmann and colleagues proposed a combination of CACS and cardiac MRI while evaluating CAD. By addition of CACS to the cardiac examination protocol, the accuracy of MRI was remarkably enhanced (94). Consecutive follow-up scorings can provide data concerning coronary calcification progression (95). Wong and colleagues (96) measuring the volume score progression and correlating that with lipid profile change assessed the efficiency of calcium scoring to evaluate the impact of lipid-lowering treatments and demonstrated that higher HDL cholesterol level was accompanied by less progression of volume score; nevertheless, could not find any evidence in favor of that CACS progression implied the low-density lipoprotein (LDL) change. Currently, many experts believe that CACS changes over the time can be considered as a method for watching the impact of lipid-lowering treatments.

Tong et al.(97) demonstrated that calcium score and left ventricular hypertrophy extent were significantly correlated in young to middle-aged African-American individuals. Women in postmenopausal age are more prone to atherosclerotic changes and CAD than premenopausal women and estrogenic hormones used to treat postmenopausal syndrome result in reduction of coronary atherosclerosis. Long term hormone replacement therapy has been shown to be effective on CACS as an indicator of CAD risk and those postmenopausal individuals utilizing estrogen for minimum 10 years revealed remarkably less CACS in comparison with those who had shorter period uses $(98,99)$. It has been shown that visceral and subcutaneous fats have different effects on cardiovascular risks. Pericardial fat is one of the visceral fat structures and as shown by Yun et al. (100) has an independent character in coronary calcification regardless of anthropometric measurements. In another study, Sabour and colleagues pointed out that there is a relationship between persistent abdominal obesity with high CACS, which suggests 
an increased risk of coronary atherosclerosis (101). Nonetheless, Shabestari and colleagues (102) have displayed that anthropometric measurements are generally more reliable than ultrasonic abdominal fat measurements in prediction of CAD. Jung et al. (103) recommended measuring the CACS for further coronary atherosclerosis assessment in cases of fatty liver and increased level of alanine aminotransferase. The ascorbic acid is a crucial antioxidant. It has been proposed that there is an association between ascorbic acid deficiency and cardiovascular disease risk (104) and hence, a study by Simon et al was performed correlating plasma ascorbic acid level and calcium score confirming their substantial correlation of a group of young men. However, this was not detected in a corresponding young female group (105).

\section{Conclusion}

As a conclusion it should be reminded that based on many data gathered over recent decades and despite presence of some controversies, the CACS advantages and disadvantages are appropriately evaluated and the correct form is its and not it's.clinical and research applications are accepted. These have led to general acceptance of calcium scoring as a standard of reference for determination of risk of cardiac events. In spite of presence of controversies, CACS has gained an acceptance to be an indicator of cardiac events risk and likely will even be more persuasive in further cardiovascular risk management in future.

\section{Acknowledgements}

There is no acknowledgment.

\section{Author's Contribution}

All the literature review and writing all the content of article was carried out by Dr. Abbas Arjmand Shabestari.

\section{Financial Disclosure}

The author has no relevant financial interest related to the material and content of this review article.

\section{Funding/Support}

Gathering data and writing of this review article are not funded or supported by any institution, university or organization.

\section{References}

1. World Health Organization Website Media Center Fact Sheet $\mathrm{N}$ 310 . 2012. Available from: http://www.who.int/mediacenter/factsheets/fs310/en.

2. Rissanen V. Coronary atherosclerosis in middle-aged and elderly Finnish men. Pathological study of coronary arteries in violent and sudden coronary deaths. Acta Pathol Microbiol Scand A. 1980;88(3):167-73.

3. Anderson HC. Calcific diseases. A concept. Arch Pathol Lab Med. 1983;107(7):341-8.
4. Sun Z, Ng KH. Multislice CT angiography in cardiac imaging. Part II: clinical applications in coronary artery disease. Singapore Med J. 2010;51(4):282-9.

5. Margolis JR, Chen JT, Kong Y, Peter RH, Behar VS, Kisslo JA. The diagnostic and prognostic significance of coronary artery calcification. A report of 800 cases. Radiology. 1980;137(3):609-16.

6. Rahimian N, Saligheh Rad H, Firouznia K, Ebrahimzadeh SA, Meysamie A, Vafaiean H, et al. Magnetic Resonance Spectroscopic Findings of Chronic Lesions in Two Subtypes of Multiple Sclerosis: Primary Progressive Versus Relapsing Remitting. Iran J Radiol. 2013;10(3):128-132.

7. Guthaner DF, Wexler L, Harell G. CT demonstration of cardiac structures. AJR Am J Roentgenol. 1979;133(1):75-81.

8. Huang CW, Tsai YF, Hsiao CY. Different MRI Signs in Predicting the Treatment Efficacy of Epidural Blood Patch in Spontaneous Intracranial Hypotension: A Case Report. Iran J Radiol. 2013;10(3):172174.

9. Masuda Y, Naito S, Uda T, Yamada Z, Morooka N, Watanabe S, et al. Clinical importance of coronary calcification detected by CT. Acta Cardiol. 1991;46(1):51-6.

10. Stanford W, Thompson BH, Weiss RM. Coronary artery calcification: clinical significance and current methods of detection. AJR Am J Roentgenol. 1993;161(6):1139-46.

11. Hsu T. Ultra-high-speed tomography: scanning electron beam CT systems. Appl Radiol.1981;10(2):86-8.

12. Bleiweis MS, Georgiou D, Brundage BH. Ultrafast CT and the cardiovascular system. The International Journal of Cardiac Imaging. 1992;8(4):289-302.

13. Özkan B, Güngör D, Yıldırım UM, Harman A, Özen O, Aytekin C. Endovascular Stent Placement of Juxtaanastomotic Stenosis in Native Arteriovenous Fistula After Unsuccessful Balloon Angioplasty. Iran J Radiol. 2013;10(3):133-139.

14. Khazan M, Azizi F, Hedayati M. A Review on Iodine Determination Methods in Salt and Biological Samples. Scimetr. 2013;1(1).

15. Agatston AS, Janowitz WR, Hildner FJ, Zusmer NR, Viamonte Jr M, Detrano R. Quantification of coronary artery calcium using ultrafast computed tomography. J Am Colle Cardiol. 1990;15(4):827832.

16. Hoff JA, Chomka EV, Krainik AJ, Daviglus M, Rich S, Kondos GT. Age and gender distributions of coronary artery calcium detected by electron beam tomography in 35,246 adults. Am J Cardiol. 2001;87(12):1335-1339.

17. Berland LL, Smith JK. Multidetector-array CT: once again, technology creates new opportunities. Radiology. 1998;209(2):327-9.

18. Leber AW, Knez A, von Ziegler F, Becker A, Nikolaou K, Paul S, et al. Quantification of obstructive and nonobstructive coronary lesions by 64-slice computed tomography: a comparative study with quantitative coronary angiography and intravascular ultrasound. J Am Coll Cardiol. 2005;46(1):147-54.

19. Ehara M, Surmely JF, Kawai M, Katoh O, Matsubara T, Terashima M, et al. Diagnostic Accuracy of 64-Slice Computed Tomography for Detecting Angiographically Significant Coronary Artery Stenosis in an Unselected Consecutive Patient Population Comparison With Conventional Invasive Angiography. Circulation Journal. 2006;70(5):564-571.

20. Shabestari AA, Abdi S, Akhlaghpoor S, Azadi M, Baharjoo $\mathrm{H}$, Pajouh MD, et al. Diagnostic performance of 64-channel multislice computed tomography in assessment of significant coronary artery disease in symptomatic subjects. Am J Cardiol. 2007;99(12):1656-61.

21. Shabestari AA, Akhlaghpoor S, Tayebivaljozi R, Fattahi Masrour F. Prevalence of Congenital Coronary Artery Anomalies and Variants in 2697 Consecutive Patients Using 64-Detector Row Coronary CTAngiography. Iran J Radiol. 2012;9(3):111-21.

22. Ohashi K, Ichikawa K, Hara M, Kawai T, Kunitomo H, Higashide R, et al. Examination of the optimal temporal resolution required for computed tomography coronary angiography. Radiol Phys Technol. 2013;6(2):453-60.

23. Almoudi M, Sun Z. Coronary artery calcium score: Re-evaluation of its predictive value for coronary artery disease. World J Cardiol. 2012;4(10):284-7.

24. Jensen CJ, Jochims M, Eberle HC, Wolf A, Nassenstein K, Forsting 


\section{Shabestari $A A$}

$\mathrm{M}$, et al. Coronary artery calcium score: influence of the reconstruction interval on cardiac risk stratification in asymptomatic patients using dual-source computed tomography. Rofo. 2011;183(3):233-7.

25. Pan Z, Pang L, Li J, Zhang H, Yang W, Ding B, et al. Achieving consistent image quality with dose optimization in 64-row multidetector computed tomography prospective ECG gated coronary calcium scoring. Int J Cardiovasc Imaging. 2011;27(4):611-7.

26. Horiguchi J, Matsuura N, Yamamoto H, Kiguchi M, Fujioka C, Kitagawa T, et al. Effect of heart rate and body mass index on the interscan and interobserver variability of coronary artery calcium scoring at prospective ECG-triggered 64-slice CT. Korean J Radiol. 2009;10(4):340-6.

27. Groen JM, Greuter MJ, Vliegenthart R, Suess C, Schmidt B, Zijlstra F, et al. Calcium scoring using 64-slice MDCT, dual source CT and EBT: a comparative phantom study. Int J Cardiovasc Imaging. 2008;24(5):547-56.

28. Ulzheimer S, Kalender WA. Assessment of calcium scoring performance in cardiac computed tomography. Eur Radiol. 2003;13(3):484-97.

29. Horiguchi J, Yamamoto H, Akiyama Y, Marukawa K, Hirai N, Ito K. Coronary artery calcium scoring using 16-MDCT and a retrospective ECG-gating reconstruction algorithm. AJR Am J Roentgenol. 2004;183(1):103-8.

30. Kopp AF, Ohnesorge B, Becker C, Schroder S, Heuschmid M, Kuttner A, et al. Reproducibility and accuracy of coronary calcium measurements with multi-detector row versus electronbeam CT. Radiology. 2002;225(1):113-9.

31. van der Bijl N, de Bruin PW, Geleijns J, Bax JJ, Schuijf JD, de Roos A, et al. Assessment of coronary artery calcium by using volumetric 320-row multi-detector computed tomography: comparison of $0.5 \mathrm{~mm}$ with $3.0 \mathrm{~mm}$ slice reconstructions. Int J Cardiovasc Imaging. 2010;26(4):473-82.

32. Detrano RC, Doherty TM, Xiang M, Kawakubo M, Labree L, Romero E, et al. Comparison of calcium scores from thick-and thin image slice-computed tomography scanning in predicting future coronary events. Am J Cardiol. 2004;93(5):624-6.

33. Hong C, Becker CR, Schoepf UJ, Ohnesorge B, Bruening R, Reiser MF. Coronary artery calcium: absolute quantification in nonenhanced and contrast-enhanced multi-detector row CT studies. Radiology. 2002;223(2):474-80.

34. Schlosser T, Hunold P, Schmermund A, Kuhl H, Waltering KU, Debatin JF, et al. Coronary artery calcium score: influence of reconstruction interval at 16-detector row CT with retrospective electrocardiographic gating. Radiology. 2004;233(2):586-9.

35. Sun Z, Choo GH, Ng KH. Coronary CT angiography: current status and continuing challenges. Br J Radiol. 2012;85(1013):495-510.

36. Kim KP, Einstein AJ, Berrington de Gonzalez A. Coronary artery calcification screening: estimated radiation dose and cancer risk. Arch Intern Med. 2009;169(13):1188-94.

37. Sun Z. Multislice CT angiography in coronary artery disease: Technical developments, radiation dose and diagnostic value. World J Cardiol. 2010;2(10):333-43.

38. Nelson JC, Kronmal RA, Carr JJ, McNitt-Gray MF, Wong ND, Loria $\mathrm{CM}$, et al. Measuring coronary calcium on CT images adjusted for attenuation differences. Radiology. 2005;235(2):403-14.

39. Muhlenbruch G, Hohl C, Das M, Wildberger JE, Suess C, Klotz E, et al. Evaluation of automated attenuation-based tube current adaptation for coronary calcium scoring in MDCT in a cohort of 262 patients. Eur Radiol. 2007;17(7):1850-7.

40. Callister TQ, Cooil B, Raya SP, Lippolis NJ, Russo DJ, Raggi P. Coronary artery disease: improved reproducibility of calcium scoring with an electron-beam CT volumetric method. Radiology. 1998;208(3):807-14.

41. Hokanson JE, MacKenzie T, Kinney G, Snell-Bergeon JK, Dabelea D, Ehrlich J, et al. Evaluating changes in coronary artery calcium: an analytic method that accounts for interscan variability. AJR Am J Roentgenol. 2004;182(5):1327-32.

42. Rumberger JA, Kaufman L. A rosetta stone for coronary calcium risk stratification: agatston, volume, and mass scores in 11,490 individuals. AJR Am J Roentgenol. 2003;181(3):743-8.

43. Nasir K, Raggi P, Rumberger JA, Braunstein JB, Post WS, Budoff
MJ, et al. Coronary artery calcium volume scores on electron beam tomography in 12,936 asymptomatic adults. Am J Cardiol. 2004;93(9):1146-9.

44. Ferencik M, Ferullo A, Achenbach S, Abbara S, Chan RC, Booth SL, et al. Coronary calcium quantification using various calibration phantoms and scoring thresholds. Invest Radiol. 2003;38(9):55966.

45. Brown ER, Kronmal RA, Bluemke DA, Guerci AD, Carr JJ, Goldin J, et al. Coronary calcium coverage score: determination, correlates, and predictive accuracy in the Multi-Ethnic Study of Atherosclerosis. Radiology. 2008;247(3):669-75.

46. Rumberger JA, Brundage BH, Rader DJ, Kondos G. Electron beam computed tomographic coronary calcium scanning: a review and guidelines for use in asymptomatic persons. Mayo Clin Proc. 1999;74(3):243-52.

47. Georgiou D, Budoff MJ, Kaufer E, Kennedy JM, Lu Bin, Brundage $\mathrm{BH}$. Screening patients with chest pain in the emergency department using electron beam tomography: a follow-up study. J Am Coll Cardiol. 2001;38(1):105-110

48. Rumberger JA, Simons DB, Fitzpatrick LA, Sheedy PF, Schwartz RS. Coronary artery calcium area by electron-beam computed tomography and coronary atherosclerotic plaque area. A histopathologic correlative study. Circulation. 1995;92(8):2157-62.

49. Guerci AD, Spadaro LA, Popma JJ, Goodman KJ, Brundage BH, Budoff $\mathrm{M}$, et al. Relation of Coronary Calcium Score by Electron Beam Computed Tomography to Arteriographic Findings in Asymptomatic and Symptomatic Adults. Am J Cardiol. 1997;79(2):128-133.

50. Rumberger JA, Sheedy PF, Breen JF, Schwartz RS. Electron Beam Computed Tomographic Coronary Calcium Score Cutpoints and Severity of Associated Angiographic Lumen Stenosis. J Am Colle Cardiol.1997;29(7):1542-1548.

51. Moser KW, O'Keefe JH, Bateman TM, McGhie IA. Coronary calcium screening in asymptomatic patients as a guide to risk factor modification and stress myocardial perfusion imaging. $J$ Nuc Cardiol. 2003;10(6):590-598.

52. Mendoza-Rodriguez V, Llerena LR, Rodriguez-de la Vega A, Rodriguez-Diaz Y. [Volume of coronary calcified plaques by computed tomography and presence of significant stenosis by coronary angiography.]. Arch Cardiol Mex. 2010;80(3):181-186.

53. Shaw LJ, Raggi P, Schisterman E, Berman DS, Callister TQ. Prog nostic value of cardiac risk factors and coronary artery calcium screening for all-cause mortality. Radiology. 2003;228(3):826-33.

54. Cheng Yiling J, Church Timothy S, Kimball Thomas E, Nichaman Milton Z, Levine Benjamin D, McGuire Darren K, et al. Comparison of coronary artery calcium detected by electron beam tomography in patients with to those without symptomatic coronary heart disease. Am J Cardiol. 2003;92(5):498-503.

55. Guerci AD, Spadaro LA, Goodman KJ, Lledo-Perez A, Newstein D, Lerner $\mathrm{G}$, et al. Comparison of electron beam computed tomography scanning and conventional risk factor assessment for the prediction of angiographic coronary artery disease. J Am Colle Cardiol.1998;32(3):673-679.

56. Shabestari AA, Akhlaghpoor S, Shadmani M, Ebrahimi M, Shakiba M, Shojaei Moghadam M. Agreement Determination between Coronary Calcium-Scoring and Coronary Stenosis Significance on CT-Angiography. Iran J Radiol. 2006;3(2):85-90.

57. Wexler L, Brundage B, Crouse J, Detrano R, Fuster V, Maddahi J, et al. Coronary artery calcification: pathophysiology, epidemiology, imaging methods, and clinical implications. A statement for health professionals from the American Heart Association. Writing Group. Circulation. 1996;94(5):1175-92.

58. Shemesh J, Apter S, Itzchak Y, Motro M. Coronary calcification compared in patients with acute versus in those with chronic coronary events by using dual-sector spiral CT. Radiology. 2003;226(2):483-8.

59. Thompson BH, Stanford W. Imaging of coronary calcium: a case for electron beam computed tomography. J Thorac Imaging. 2001;16(1):8-15.

60. Knez A, Becker A, Leber A, White C, Becker CR, Reiser MF, et al. Relation of coronary calcium scores by electron beam tomography to obstructive disease in 2,115 symptomatic patients. Am J Cardiol. 
2004;93(9):1150-2.

61. Ergun E, Kosar P, Ozturk C, Basbay E, Koc F, Kosar U. Prevalence and extent of coronary artery disease determined by 64-slice CTA in patients with zero coronary calcium score. Int J Cardiovasc Imaging. 2011;27(3):451-8

62. Uretsky S, Rozanski A, Singh P, Supariwala A, Atluri P, Bangalore S, et al. The presence, characterization and prognosis of coronary plaques among patients with zero coronary calcium scores. Int J Cardiovasc Imaging. 2011;27(6):805-12.

63. Church TS, Levine BD, McGuire DK, Lamonte MJ, Fitzgerald SJ, Cheng YJ, et al. Coronary artery calcium score, risk factors, and incident coronary heart disease events. Atherosclerosis. 2007;190(1):224-31

64. Grayburn PA. Interpreting the coronary-artery calcium score. $N$ Engl J Med. 2012;366(4):294-6.

65. Johnson KM, Dowe DA, Brink JA. Traditional clinical risk assessment tools do not accurately predict coronary atherosclerotic plaque burden: a CT angiography study. AJR Am J Roentgenol. 2009;192(1):235-43.

66. Budoff MJ, Nasir K, McClelland RL, Detrano R, Wong N, Blumenthal RS, et al. Coronary calcium predicts events better with absolute calcium scores than age-sex-race/ethnicity percentiles: MESA (Multi-Ethnic Study of Atherosclerosis). J Am Coll Cardiol. 2009;53(4):345-52.

67. Kwon SW, Kim YJ, Shim J, Sung JM, Han ME, Kang DW, et al. Coronary artery calcium scoring does not add prognostic value to standard 64-section CT angiography protocol in low-risk patients suspected of having coronary artery disease. Radiology. 2011;259(1):92-9.

68. van Werkhoven JM, de Boer SM, Schuijf JD, Cademartiri F, Maffei E, Jukema JW, et al. Impact of clinical presentation and pretest likelihood on the relation between calcium score and computed tomographic coronary angiography. Am J Cardiol. 2010;106(12):1675-9.

69. Meng L, Cui L, Cheng Y, Wu X, Tang Y, Wang Y, et al. Effect of heart rate and coronary calcification on the diagnostic accuracy of the dual-source CT coronary angiography in patients with suspected coronary artery disease. Korean J Radiol. 2009;10(4):347-54.

70. Asci CCT, C. M. R. Guideline Working Group , Tsai IC, Choi BW, Chan C, Jinzaki M, et al. ASCI 2010 appropriateness criteria for cardiac computed tomography: a report of the Asian Society of Cardiovascular Imaging Cardiac Computed Tomography and Cardiac Magnetic Resonance Imaging Guideline Working Group. Int J Cardiovasc Imaging. 2010;26 Suppl 1:1-15.

71. Akram K, O'Donnell RE, King S, Superko HR, Agatston A, Voros S Influence of symptomatic status on the prevalence of obstructive coronary artery disease in patients with zero calcium score. Atherosclerosis. 2009;203(2):533-7.

72. Cademartiri F, Maffei E, Palumbo A, Martini C, Seitun S, Tedeschi C, et al. Diagnostic accuracy of computed tomography coronary angiography in patients with a zero calcium score. Eur Radiol. 2010;20(1):81-7.

73. Gottlieb I, Miller JM, Arbab-Zadeh A, Dewey M, Clouse ME, Sara L, et al. The absence of coronary calcification does not exclude obstructive coronary artery disease or the need for revascularization in patients referred for conventional coronary angiography. JAm Coll Cardiol. 2010;55(7):627-34

74. Esteves FP, Khan A, Correia LC, Nye JA, Halkar RK, Schuster DM, et al. Absent coronary artery calcium excludes inducible myocardial ischemia on computed tomography/positron emission tomography. Int J Cardiol. 2011;147(3):424-7.

75. Sosnowski M, Pysz P, Szymanski L, Gola A, Tendera M. Negative calcium score and the presence of obstructive coronary lesions in patients with intermediate CAD probability. Int $J$ Cardiol. 2011;148(1):e16-8.

76. Alqarqaz M, Zaidan M, Al-Mallah MH. Prevalence and predictors of atherosclerosis in symptomatic patients with zero calcium score. Acad Radiol. 2011;18(11):1437-41.

77. Villines TC, Hulten EA, Shaw LJ, Goyal M, Dunning A, Achenbach $S$, et al. Prevalence and severity of coronary artery disease and adverse events among symptomatic patients with coronary artery calcification scores of zero undergoing coronary computed tomography angiography: results from the CONFIRM (Coronary CT Angiography Evaluation for Clinical Outcomes: An International Multicenter) registry.J Am Coll Cardiol. 2011;58(24):2533-40.

78. Chen CK, Kuo YS, Liu CA, Sheu MH, Chang HT, Chen CW, et al. Frequency and risk factors associated with atherosclerotic plaques in patients with a zero coronary artery calcium score. J Chin Med Assoc. 2012;75(1):10-5.

79. Morita H, Fujimoto S, Kondo T, Arai T, Sekine T, Matsutani H, et al. Prevalence of computed tomographic angiography-verified high-risk plaques and significant luminal stenosis in patients with zero coronary calcium score. Int J Cardiol. 2012;158(2):272-8.

80. Kim YJ, Hur J, Lee HJ, Chang HJ, Nam JE, Hong YJ, et al. Meaning of zero coronary calcium score in symptomatic patients referred for coronary computed tomographic angiography. Eur Heart J Cardiovasc Imaging. 2012;13(9):776-85.

81. Meyer M, Henzler T, Fink C, Vliegenthart R, Barraza JM, Jr, Nance JW, Jr, et al. Impact of coronary calcium score on the prevalence of coronary artery stenosis on dual source CT coronary angiography in caucasian patients with an intermediate risk. Acad Radiol. 2012;19(11):1316-23.

82. Buyukterzi M, Turkvatan A, Buyukterzi Z. Frequency and extent of coronary atherosclerotic plaques in patients with a coronary artery calcium score of zero: assessment with CT angiography. Diagn Interv Radiol. 2013;19(2):111-8.

83. Cho I, Suh JW, Chang HJ, Kim KI, Jeon EJ, Choi SI, et al. Prevalence and prognostic implication of non-calcified plaque in asymptomatic population with coronary artery calcium score of zero. Korean Circ J. 2013;43(3):154-60.

84. Lee MS, Chun EJ, Kim KJ, Kim JA, Yoo JY, Choi SI. Asymptomatic subjects with zero coronary calcium score: coronary CT angiographic features of plaques in event-prone patients. Int J Cardiovasc Imaging. 2013;29 Suppl 1:29-36.

85. Mouden M, Timmer JR, Reiffers S, Oostdijk AH, Knollema S, Ottervanger JP, et al. Coronary artery calcium scoring to exclude flowlimiting coronary artery disease in symptomatic stable patients at low or intermediate risk. Radiology. 2013;269(1):77-83.

86. Taylor AJ, Cerqueira M, Hodgson JM, Mark D, Min J, O'Gara P, et al. ACCF/SCCT/ACR/AHA/ASE/ASNC/NASCI/SCAI/SCMR 2010 appropriate use criteria for cardiac computed tomography. A report of the American College of Cardiology Foundation Appropriate Use Criteria Task Force, the Society of Cardiovascular Computed Tomography, the American College of Radiology, the American Heart Association, the American Society of Echocardiography, the American Society of Nuclear Cardiology, the North American Society for Cardiovascular Imaging, the Society for Cardiovascular Angiography and Interventions, and the Society for Cardiovascular Magnetic Resonance. JAm Coll Cardiol. 2010;56(22):186494.

87. Otton JM, Yu CY, McCrohon J, Sammel N, Feneley M. Accuracy and clinical outcomes of computed tomography coronary angiography in the presence of a high coronary calcium score. Heart Lung Circ. 2013;22(12):980-6.

88. Ahn SJ, Kang DK, Sun JS, Yoon MH. Accuracy and predictive value of coronary computed tomography angiography for the detection of obstructive coronary heart disease in patients with an agatston calcium score above 400. J Comput Assist Tomogr. 2013;37(3):387-94.

89. Otton J, Feneley M, Sammel N, McCrohon J. The accuracy of cardiovascular computed tomography in the presence of a high coronary calcium score may be underestimated. J Am Coll Cardiol. 2012;60(7):642.

90. Arjmand Shabestari A, Pourghorban R, Tehrai M, Pouraliakbar $\mathrm{H}$, Faghihi Langroudi T, Bakhshandeh $\mathrm{H}$, et al. Comparison of aortic root dimension changes during cardiac cycle between the patients with and without aortic valve calcification using ECGgated 64-slice and dual-source 256-slice computed tomography scanners: results of a multicenter study. Int J Cardiovasc Imaging. 2013;29(6):1391-400.

91. Elias-Smale SE, Proenca RV, Koller MT, Kavousi M, van Rooij FJ, Hunink MG, et al. Coronary calcium score improves classification of coronary heart disease risk in the elderly: the Rotterdam study. J Am Coll Cardiol. 2010;56(17):1407-14. 


\section{Shabestari AA}

92. Sirineni GK, Raggi P, Shaw LJ, Stillman AE. Calculation of coronary age using calcium scores in multiple ethnicities. Int J Cardiovasc Imaging. 2008;24(1):107-11.

93. Colletti PM, Dustin LD, Wong ND, Shriki JE, Kawakubo M, Azen SP, et al. Does coronary calcium score predict future cardiac function? Association of subclinical atherosclerosis with left ventricular systolic and diastolic dysfunction at MR imaging in an elderly cohort. Radiology. 2010;257(1):64-70.

94. Stolzmann P, Alkadhi H, Scheffel H, Plass A, Leschka S, Falk V, et al. Combining cardiac magnetic resonance and computed tomography coronary calcium scoring: added value for the assessment of morphological coronary disease? Int J Cardiovasc Imaging. 2011;27(7):969-77.

95. Raggi Paolo, Cooil Bruce, Shaw Leslee J, Aboulhson Jamil, Takasu Junichiro, Budoff Matthew, et al. Progression of coronary calcium on serial electron beam tomographic scanning is greater in patients with future myocardial infarction. Am Journal Cardiol. 2003;92(7):827-829.

96. Wong ND, Kawakubo M, LaBree L, Azen SP, Xiang M, Detrano R. Relation of coronary calcium progression and control of lipids according to National Cholesterol Education Program guidelines. Am J Cardiol. 2004;94(4):431-6. 


\section{Shabestari AA}

97. Tong W, Lima JA, Lai H, Celentano DD, Dai S, Lai S. Relation of coronary artery calcium to left ventricular mass in African-Americans. Am J Cardiol. 2004;93(4):490-2.

98. Sullivan JM, Vander Zwaag R, Lemp GF, Hughes JP, Maddock V, Kroetz FW, et al. Postmenopausal estrogen use and coronary atherosclerosis. Ann Intern Med.1988;108(3):358-63.

99. Barrett-Connor E, Laughlin GA. Hormone therapy and coronary artery calcification in asymptomatic postmenopausal women: the Rancho Bernardo Study. Menopause. 2005;12(1):40-8.

100. Yun CH, Lin TY, Wu YJ, Liu CC, Kuo JY, Yeh HI, et al. Pericardial and thoracic peri-aortic adipose tissues contribute to systemic inflammation and calcified coronary atherosclerosis independent of body fat composition, anthropometric measures and traditional cardiovascular risks. Eur J Radiol. 2012;81(4):749-56.

101. Sabour S, Grobbee DE, Prokop M, van der Schouw YT, Bots ML.
Change in abdominal obesity and risk of coronary calcification. J Epidemiol Community Health. 2011;65(3):287-8.

102. Shabestari AA, Bahrami-Motlagh H, Hosseinpanah F, Heidari K. Abdominal fat sonographic measurement compared to anthropometric indices for predicting the presence of coronary artery disease. J Ultrasound Med. 2013;32(11):1957-65.

103. Jung DH, Lee YJ, Ahn HY, Shim JY, Lee HR. Relationship of hepatic steatosis and alanine aminotransferase with coronary calcification. Clin Chem Lab Med. 2010;48(12):1829-34.

104. Simon JA. Vitamin C and cardiovascular disease: a review. J Am Coll Nutr. 1992;11(2):107-25.

105. Simon JA, Murtaugh MA, Gross MD, Loria CM, Hulley SB, Jacobs DR, Jr. Relation of ascorbic acid to coronary artery calcium: the Coronary Artery Risk Development in Young Adults Study. Am J Epidemiol. 2004;159(6):581-8. 